\title{
Performance of a Novel Research-Use-Only Secretoneurin ELISA in Patients with Suspected Acute Coronary Syndrome: Comparison with an Established Secretoneurin Radioimmunoassay
}

\author{
Peder L. Myhre ${ }^{a, b}$ Anett H. Ottesen ${ }^{c, d} \quad$ Arne L. Faaren $^{e} \quad$ Sjur H. Tveit ${ }^{a, b}$ \\ Jon Springett ${ }^{f} \quad$ Johanna Pyylampi $^{g} \quad$ Mats Stridsberg $^{\text {h }}$ Geir Christensen $^{b, d}$ \\ Arne Didrik Høiseth $^{a}$ Torbjørn Omland ${ }^{a, b}$ Helge Røsjø ${ }^{b, c}$

\begin{abstract}
aDivision of Medicine, Department of Cardiology, Akershus University Hospital, Lørenskog, Norway; bInstitute of Clinical Medicine, University of Oslo, Oslo, Norway; ' Division for Research and Innovation, Akershus University

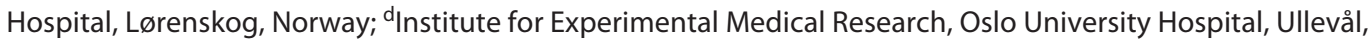

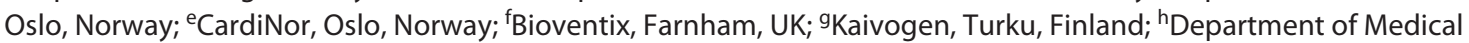 \\ Sciences, Uppsala University, Uppsala, Sweden
}

\section{Keywords}

Secretoneurin · Enzyme-linked immunosorbent assay ·

Acute coronary syndrome

\begin{abstract}
Background: Circulating secretoneurin (SN) concentrations, as measured by established radioimmunoassay (RIA), risk stratify patients with cardiovascular disease. We now report data for a recently developed research-use-only SN enzymelinked immunosorbent assay (ELISA) in patients with suspected acute coronary syndrome (ACS). Methods: SN ELISA was developed according to industry standards and tested in 401 unselected chest pain patients. Blood samples were drawn $<24 \mathrm{~h}$ from admission, and we adjudicated all hospitalizations as ACS or non-ACS. The mean follow-up was 6.2 years. Results: SN ELISA with 2 monoclonal sheep anti-SN antibodies has a measuring range of $10-250 \mathrm{pmol} / \mathrm{L}$ and demonstrates excellent analytical precision and accuracy
\end{abstract}

across the range of $\mathrm{SN}$ concentrations. $\mathrm{SN}$ measured by ELI$\mathrm{SA}$ and RIA correlated in the chest pain patients: $\mathrm{rho}=0.39$, $p<0.001$. SN concentrations were higher in ACS patients $(n=161[40 \%])$ than in non-ACS patients $(n=240)$ for both assays, with an area under the curve (AUC) of $0.66(95 \% \mathrm{Cl}$ : $0.61-0.71)$ for ELISA and 0.59 (0.54-0.65) for RIA. SN concentrations were also higher in nonsurvivors $(n=65$ [16\%]) than survivors, with an AUC of $0.72(0.65-0.79)$ for ELISA versus $0.64(0.56-0.72)$ for RIA, $p=0.007$, for difference between assays. Adjusting for age, sex, blood pressure, previous myocardial infarction, atrial fibrillation, and heart failure in multivariable analysis, SN concentrations as measured by ELISA, but not RIA, remained associated with mortality, with a hazard ratio of 1.71 (1.03-2.84), $p=0.038$. Conclusions: The novel SN ELISA has excellent performance, higher AUC for diagnosis, and superior prognostic accuracy compared to the established RIA in chest pain patients.

(C) 2021 The Author(s)

Published by S. Karger AG, Basel karger@karger.com

www.karger.com/crd

Karger ${ }^{\prime \prime}$

BOPEN ACCESS
(C) 2021 The Author(s)

Published by S. Karger AG, Basel

This is an Open Access article licensed under the Creative Commons Attribution-NonCommercial-4.0 International License (CC BY-NC) (http://www.karger.com/Services/OpenAccessLicense), applicable to the online version of the article only. Usage and distribution for commercial purposes requires written permission.
Correspondence to:

Peder L. Myhre, p.l.myhre@medisin.uio.no

Helge Røsjø, helge.rosjo@medisin.uio.no 


\section{Introduction}

Secretoneurin (SN) is a biologically active fragment of secretogranin II [1], which belongs to the granin protein family [2]. SN directly influences cardiomyocyte $\mathrm{Ca}^{2+}$ handling, and circulating SN concentrations provide prognostic information in patients with acute heart failure [1], cardiovascular-related acute respiratory failure [3], sepsis [4], and ventricular arrhythmia-induced cardiac arrest [1]. Thus, SN represents a promising cardiovascular biomarker that could complement established risk indices and biomarkers.

The pathophysiology reflected by $\mathrm{SN}$ as a biomarker is not clear. Early reports suggested that $\mathrm{SN}$ is linked to cardiomyocyte $\mathrm{Ca}^{2+}$ handling [5], but $\mathrm{SN}$ could also be influenced by additional cardiac pathophysiology. Work in experimental models has demonstrated enhanced SN production by cellular hypoxia [6] and increased myocardial SN concentrations after coronary artery ligation in mice [7]. However, whether myocardial ischemia increases circulating $\mathrm{SN}$ concentrations in patients is not known. Moreover, the performance of SN as a prognostic biomarker in patients with suspected acute coronary syndrome (ACS) has not been explored.

The potential of SN as a cardiac biomarker in clinical practice is dependent on an easily available and robust method for SN quantification. To this date, SN has been measured by in-house radioimmunoassays (RIAs) in studies demonstrating improved risk stratification by SN measurements in patients with myocardial dysfunction $[1,3,4,8-11]$. However, as RIA is time consuming and requires special laboratories due to radiation, there is a need for novel SN assays on platforms more practical for clinical laboratories. Hence, we have recently developed a research-use-only enzyme-linked immunosorbent assay (ELISA) for SN measurements with 2 monoclonal sheep anti-SN antibodies that detect the 33-amino acidlong SN peptide. Accordingly, in this work, we hypothesized that the novel SN ELISA would provide comparable or superior performance to the established in-house RIA for diagnosis and risk stratification in unselected patients hospitalized with chest pain.

\section{Materials and Methods}

\section{Study Design}

The Akershus Cardiac Examination (ACE) 3 Study was a prospective, single-center study aiming to assess established and novel cardiovascular biomarkers in unselected patients with chest pain. The study was conducted at the Akershus University Hospital from
June 2009 to December 2010. We included all patients aged $>18$ years with an informed consent, admitted to the emergency department, daytime on weekdays, with chest pain as the primary distress. Exclusion criteria were disseminated malignant disease, acute myocardial infarction, coronary intervention or major surgery during last 2 weeks, inadequate blood sampling, and dementia or other cognitive dysfunction that made informed consent impossible. The study was approved by the Regional Ethics Committee and conducted according to the Declaration of Helsinki.

\section{Data Collection}

We collected demographic data, medical history, detailed information on the character and duration of chest pain, vital signs, and medication on hospital admission, during the stay and at discharge. In addition, we obtained clinical information directly from the attending physician in the emergency department with a standardized questionnaire. These included findings from the initial clinical examination, recordings of the electrocardiogram (ECG), and cardiac troponin $\mathrm{T}$ ( $\mathrm{cTnT}$ ) concentrations. Assessment of ECGs for indices of acute ischemia was performed, as previously reported [12], based on ESC guidelines [13].

\section{Adjudication of Diagnosis and Follow-Up}

The final diagnosis of the index hospitalization was determined by 2 independent senior physicians, who reviewed all medical records during the index hospitalization and follow-up. The reason for chest pain was classified as either ACS or non-ACS. Discrepancy in the adjudication committee was only present in $4 \%$ of the cases, and it was resolved by consensus. Diagnosis of acute myocardial infarction was based on criteria defined by the Third Universal Definition of Myocardial Infarction [14] with the use of serial cTnT measurements as part of the clinical routine in the central laboratory (i.e., rise and/or fall of $\mathrm{cTnT}$ with at least one value above the 99th percentile upper reference limit of $14 \mathrm{ng} / \mathrm{L}$ ). We based the diagnosis of unstable angina pectoris on clinical presentation, ECG findings, available examinations (exercise ECG, myocardial scintigraphy, and echocardiography), and coronary angiography (invasive and noninvasive by computer tomography) as recommended by guidelines [15]. The survival status was obtained on December 1, 2016 from electronic hospital records, which are updated with Statistics Norway on a monthly basis.

Biospecimens Collection and Standard Biochemical Analysis

Blood sampling was conducted within the first $24 \mathrm{~h}$ of hospital admission in available patients and at day 2 and at the day of discharge. Biospecimens were handled according to a standardized protocol and put on ice prior to centrifugation and later aliquoted into $500 \mu \mathrm{L}$ cryovials. Samples were kept frozen at $-80^{\circ} \mathrm{C}$ until thawed for biomarker measurements. We collected information on standard biochemical variables from routine measurements, including creatinine, and we calculated the estimated glomerular filtration rate (eGFR) by the Chronic Kidney Disease Epidemiology Collaboration (CKD-EPI) equation [16]. In contrast, we used the study-specific biobank for analysis of cTnT and N-terminal pro-Btype natriuretic peptide (NT-proBNP) concentrations. cTnT was measured in the clinical routine and biobank samples by a highsensitivity (hs) assay (Gen 5 STAT) with a level of blank at $3 \mathrm{ng} / \mathrm{L}$, level of detection at $5 \mathrm{ng} / \mathrm{L}$, and a 99th percentile upper reference limit of $14 \mathrm{ng} / \mathrm{L}$. The coefficient of variation (CV) was $2.5 \%$ at 68 and $3.0 \%$ at $925 \mathrm{ng} / \mathrm{L}(n \geq 24)$. Unmeasurable cTnT concentrations 


\section{Epitope maps: SN (Sgll 154-186)}

i)

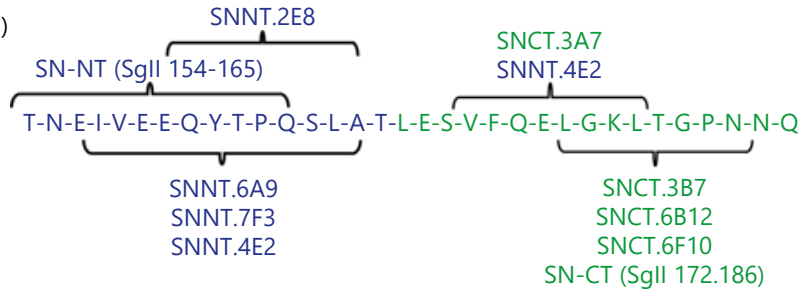

ii)

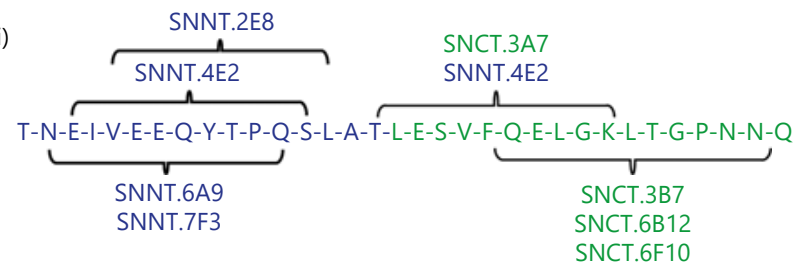

Antibodies from Bioventix SNNT.2E8 SNCT.3A7 SNNT.4E2 SNCT.3B7 SNNT.6A9 SNCT.6B12

a SNNT.7F3 SNCT.6F10
SPOT peptide arrays

T-N-E-I-V-E-E-Q-Y-T-P-Q-S-L-A-T-L-E-S-V-F-Q-E-L-G-K-L-T-G-P-N-N-Q

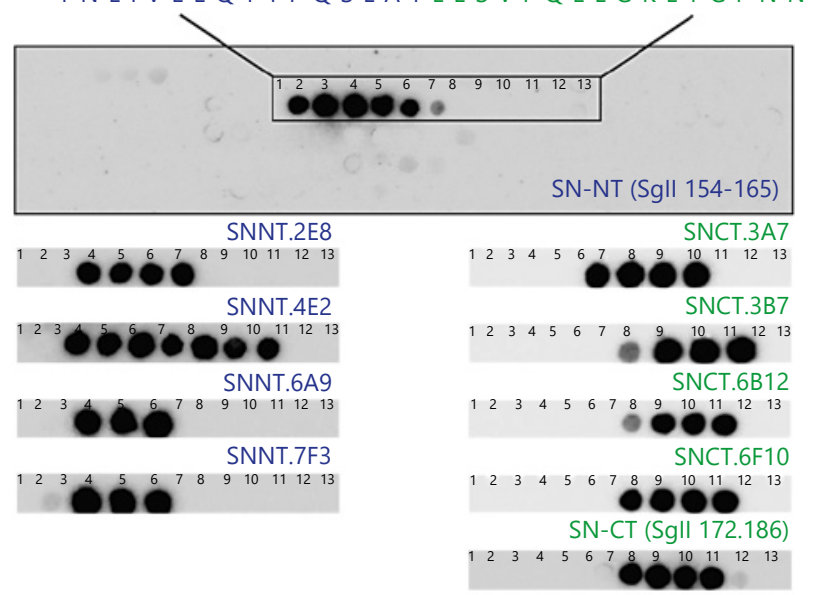

\begin{tabular}{|c|c|}
\hline Spot\#Sgll & Spot\#SN \\
\hline 42 & 1 \\
\hline 43 & 2 \\
\hline 44 & 3 \\
\hline 45 & 4 \\
\hline 46 & 5 \\
\hline 47 & 6 \\
\hline 48 & 7 \\
\hline 49 & 8 \\
\hline 50 & 9 \\
\hline 51 & 10 \\
\hline 52 & 11 \\
\hline 53 & 12 \\
\hline 54 & 13 \\
\hline
\end{tabular}

Sequence

Q-F-P-P-M-Y-E-E-N-S-R-D-N-P-F-K-R-T-N-E $M-Y-E-E-N-S-R-D-N-P-F-K-R-T-N-E-I-V-E-E$ N-S-R-D-N-P-F-K-R-T-N-E-I-V-E-E-Q-Y-T-P N-P-F-K-R-T-N-E-I-V-E-E-Q-Y-T-P-Q-S-L-A R-T-N-E-I-V-E-E-Q-Y-T-P-Q-S-L-A-T-L-E-S I-V-E-E-Q-Y-T-P-Q-S-L-A-T-L-E-S-V-F-Q-E Q-Y-T-P-Q-S-L-A-T-L-E-S-V-F-Q-E-L-G-K-L Q-S-L-A-T-L-E-S-V-F-Q-E-L-G-K-L-T-G-P-N T-L-E-S-V-F-O-E-L-G-K-L-T-G-P-N-N-O-K-R V-F-Q-E-L-G-K-L-T-G-P-N-N-Q-K-R-E-R-M-D L-G-K-L-T-G-P-N-N-O-K-R-E-R-M-D-E-E-Q-K T-G-P-N-N-Q-K-R-E-R-M-D-E-E-Q-K-L-Y-T-D N-Q-K-R-E-R-M-D-E-E-Q-K-L-Y-T-D-D-E-D-D

Fig. 1. Epitope mapping of SN antibodies. a Epitope mapping of SN antibodies performed at (i) AHUS and (ii) Bioventix. The raw data from the epitope mapping performed at AHUS are shown in $\mathbf{b}$. Amino acids in bold constitutes the core epitope that is relevant for anti-SN binding ( $n=2,2$ independent SPOT peptide arrays). SN, secretoneurin.

were reported as $3 \mathrm{ng} / \mathrm{L}$. NT-proBNP concentrations were measured using the proBNP II assay. All analyses were performed on the Elecsys platform (Cobas 8000), and the assays and platforms were produced by Roche Diagnostics (Rotkreuz, Switzerland).

\section{Assays for SN Quantification}

SN RIA

SN concentrations were measured by both the in-house SN RIA and SN ELISA. Quantification with SN RIA was performed in 2011-2012 at the Uppsala University Hospital, Sweden, as previously described [17-19]. The RIA has a reported limit of detection for SN in plasma of $50 \mathrm{pmol} / \mathrm{L}$, and the assay has a reported $\mathrm{CV}$ of $9 \%$ in the lower range (110 pmol/L) and $4 \%$ in the upper range $(380$ $\mathrm{pmol} / \mathrm{L})$. Performing the RIA requires days for completion due to the need for incubation prior to SN quantification.

\section{SN ELISA}

SN was also quantitated by a novel research-use-only SN ELISA (CardiNor AS, Oslo, Norway). SN ELISA is a sandwich assay that uses primary and secondary monoclonal sheep anti-SN antibodies
(Bioventix, Farnham, UK) with custom-made HRP-conjugated anti-SN antibody for detection (Fleet Bioprocessing, UK). SN ELISA uses a streptavidin-coated microplate, and the biotinylated $\mathrm{SN}$-specific primary antibody (SNNT.2E8) is added to the wells of the micro plate. After washing, the system requires the addition of calibrators, controls, and samples (all diluted 1:10) in duplicate with SN molecules bound by the immobilized primary antibody. Unbound substances are removed, and an HRP-conjugated specific second SN antibody (SNCT.3B7) is added and incubated. The unbound conjugate is removed by washing, and an HRP substrate is added. Finally, the stop solution is added, and the intensity of color developed is measured. A standard linear regression method is used to calculate $\mathrm{SN}$ concentrations. We selected antibodies after detailed assessment of antibody performance, including epitope mapping with a system established in our research group [20]. For epitope mapping, we used SPOT peptide arrays to assess where the antibodies bind in the full secretogranin II sequence, which was synthesized as 20 -mer overlapping peptides with a 4 -amino acid offset on cellulose membranes using a MultiPep automated peptide synthesizer (INTAVIS, Bioanalytical Instruments AG, Co- 
logne, Germany) [21]. We included a large number of candidate antibodies for mapping, including the polyclonal rabbit antibodies used for the SN RIA, with results presented in Figure 1. We also validated the results from epitope mapping with testing of antibody affinity for SN by a competition/inhibition ELISA method (see online suppl. Fig. 1; for all online suppl. material describing details of the methodology for the antibody affinity testing, see www.karger.com/doi/10.1159/000517444). The SN ELISA method does not require a pre-analytic incubation period, and the total time for analyzing a batch is $3 \mathrm{~h}$.

\section{Statistical Analysis}

Baseline characteristics are presented as proportions for categorical variables, mean \pm standard deviation for normally distributed continuous variables, and medians (quartile [Q] 1-3) for skewed continuous variables. These characteristics were compared across quartiles of $\mathrm{SN}$ using parametric and nonparametric tests, as appropriate. We transformed SN, NT-proBNP, and hs-TnT by the natural logarithm prior to regression analysis due to a rightskewed distribution. Predictors of SN measured by both assays were assessed by multivariable linear regression and correlations by the Spearman rank correlation. The associations between SN measured by the RIA and ELISA and ACS were evaluated by logistic regression analysis and by calculating receiver-operating statistics area under the curve. We evaluated the association between SN measured by both assays and all-cause mortality by Cox proportional hazard regression analysis in (1) unadjusted analysis; (2) after adjustment for age and sex; (3) after additional adjustment for hypertension, atrial fibrillation, heart failure, and previous myocardial infarction; and (4) after additional adjustment for eGFR and hs-TnT. The prognostic accuracies of SN measured by the RIA and ELISA were also calculated and compared by Harrell's C-statistics. We used the Kaplan-Meier method to present survival plots according to quartiles of SN measured by each assay. Statistical analysis was performed using STATA software v15.1 (StatCorp, College Station, TX, USA). A 2 -sided $p$ value $<0.05$ was considered significant.

\section{Results}

\section{Antibody Testing and SN ELISA Characterization}

All of the antibodies tested were found to be specific for the $\mathrm{SN}$ region of secretogranin II during epitope mapping (Fig. 1). The antibodies SN-NT (SgII 154-165), SNNT.2E8, SNNT.6A9, and SNNT.7F3 bind to the Nterminal end of SN, while SN-CT (SgII 172-186), SNCT.3A7, SNCT.3B7, SNCT.6B12, and SNCT.6F10 bind to the $\mathrm{C}$ terminal of SN. The antibody SNNT.4E2 seems to bind both the $\mathrm{N}$-terminal and the $\mathrm{C}$-terminal end of SN. Testing of antibody affinity demonstrated similar binding properties for the candidate antibodies as identified during epitope mapping (online suppl. Fig. 1).

Testing of SN sandwich ELISA with SNNT.2E8 and SNCT.3B7 demonstrated that the range of SN concentration that can be detected by SN ELISA is $10-250 \mathrm{pmol} / \mathrm{L}$ (online suppl. Fig. 2), showing linearity across the measuring range (see online suppl. Tables 1, 2; and online suppl. Fig. 3). The level of quantification was $2.3 \mathrm{pmol} / \mathrm{L}$ (estimated as mean value of buffer blank plus 10 standard deviations of the mean expressed in analyte concentration) is well below the measuring range (details in online suppl.). The SN ELISA demonstrates excellent intra-assay and inter-assay CVs, $<5$ and $<7 \%$, respectively (online suppl. Table 3).

\section{Patient Characteristics in the ACE 3 Study}

Among the 401 patients included in the study, the mean age was $61 \pm 15$ years and 191 (48\%) were female. The prevalence of cardiovascular comorbidities was 134 (34\%) for hypertension, 44 (11\%) for diabetes mellitus, 86 $(22 \%)$ with a previous myocardial infarction, 60 (15\%) for atrial fibrillation, and 21 (5\%) with heart failure. Median (Q1-Q3) SN concentrations in the total population were $127(110-148) \mathrm{pmol} / \mathrm{L}$ as measured by the RIA and 30.1 (25.3-36.8) $\mathrm{pmol} / \mathrm{L}$ as measured by ELISA. The correlation coefficient between SN measured by the RIA and ELISA was $0.39, p<0.001$. Baseline characteristics according to quartiles of SN ELISA are presented in Table 1. A higher quartile of SN ELISA was associated with older age, lower BMI, higher heart rate, more frequent ischemia on ECG, comorbidities and cardiovascular preventive medications, higher hs-TnT and NT-proBNP concentrations, and lower eGFR.

SN concentrations, as measured by both the RIA and ELISA, correlated with several important cardiovascular risk factors and biomarkers, including hs-TnT and NTproBNP (online suppl. Table 4). Still, in multivariable linear regression analysis, only eGFR $(t=-8.0, p<0.001)$ and NT-proBNP $(t=3.9, p<0.001)$ remained associated with SN ELISA, and these explained $29 \%$ of the variation in SN ELISA $\left(r^{2}=0.29\right)$. Analogously, eGFR $(t=-4.5$, $p<0.001)$, NT-proBNP $(t=3.4, p=0.001)$, and age $(t=$ $-2.7, p=0.008)$ were associated with SN RIA in multivariable models, and these explained $10 \%$ of the variation in SN ELISA $\left(r^{2}=0.10\right)$.

\section{$S N$ in Diagnosing ACS}

ACS was diagnosed in 161 of the 401 chest pain patients included in this study ( $40 \%$ of the total population). Among the patients classified with ACS, 73 patients were diagnosed with acute myocardial infarction (45\%) and 88 patients with unstable angina pectoris.

Patients with ACS were older, had more comorbidities, and more frequently used preventive medications compared to patients without ACS. A diagnosis of ACS 
Table 1. Baseline characteristics by quartiles of SN measured by ELISA

\begin{tabular}{|c|c|c|c|c|c|}
\hline & SN Q1 $(n=101)$ & $\mathrm{SN} \mathrm{Q} 2(n=101)$ & SN Q3 $(n=102)$ & SN Q4 $(n=98)$ & $p$ \\
\hline $\mathrm{SN}$ range, $\mathrm{pmol} / \mathrm{L}$ & $13.4-25.2$ & $25.3-30.1$ & $30.2-36.8$ & $\begin{array}{l}36.9-97.6 \\
(+840 \text { in one outlier })\end{array}$ & \\
\hline Male sex, $n(\%)$ & $60(60.0)$ & $52(51.5)$ & $55(53.9)$ & $43(43.9)$ & 0.042 \\
\hline BMI & $30.4 \pm 5.9$ & $27.2 \pm 4.0$ & $26.7 \pm 4.2$ & $25.0 \pm 4.1$ & $<0.001$ \\
\hline Systolic blood pressure & $148 \pm 24$ & $151 \pm 26$ & $149 \pm 25$ & $147 \pm 31$ & 0.67 \\
\hline ECG acute ischemia, $n(\%)$ & $13(13.0)$ & $16(15.8)$ & $19(18.6)$ & $26(26.5)$ & 0.013 \\
\hline ECG QRS width & $101 \pm 16$ & $99 \pm 18$ & $99 \pm 20$ & $102 \pm 24$ & 0.71 \\
\hline \multicolumn{6}{|l|}{ Medical history, $n(\%)$} \\
\hline Hypertension & $26(26.0)$ & $31(31.0)$ & $33(32.4)$ & $44(44.9)$ & 0.006 \\
\hline Heart failure & $0(0.0)$ & $6(6.0)$ & $4(3.9)$ & $11(11.2)$ & 0.002 \\
\hline Atrial fibrillation & $12(12.0)$ & $11(11.0)$ & $10(9.8)$ & $27(27.6)$ & 0.005 \\
\hline Statin & $37(37.0)$ & $37(36.6)$ & $44(43.1)$ & $56(57.1)$ & 0.003 \\
\hline RAS inhibitors & $26(26.0)$ & $24(23.8)$ & $34(33.3)$ & $47(48.0)$ & $<0.001$ \\
\hline Beta-blocker & $28(28.0)$ & $31(30.7)$ & $42(41.2)$ & $56(57.1)$ & $<0.001$ \\
\hline Diuretics & $5(5.0)$ & $7(6.9)$ & $9(8.8)$ & $31(31.6)$ & $<0.001$ \\
\hline \multicolumn{6}{|l|}{ Laboratory } \\
\hline Hemoglobin, g/dL & $14.4(13.2,15.0)$ & $14.0(13.1,14.8)$ & $13.8(12.7,15.2)$ & $13.4(12.1,14.7)$ & $<0.001$ \\
\hline C-reactive protein, $\mathrm{mg} / \mathrm{L}$ & $5(5,8)$ & $5(5,5)$ & $5(5,5)$ & $5(5,10)$ & 0.29 \\
\hline $\mathrm{eGFR}, \mathrm{mL} / \mathrm{min} / 1.73 \mathrm{~m}^{2}$ & $92(81,102)$ & $88(76,100)$ & $81(68,95)$ & $59(47,83)$ & $<0.001$ \\
\hline Total cholesterol, $\mathrm{mmol} / \mathrm{L}$ & $5.1(4.4,5.9)$ & $5.4(4.3,6.1)$ & $5.0(4.1,6.1)$ & $5.1(4.3,5.8)$ & 0.57 \\
\hline LDL cholesterol, $\mathrm{mmol} / \mathrm{L}$ & $3.1(2.3,3.8)$ & $3.1(2.3,3.9)$ & $2.8(2.2,3.8)$ & $2.8(2.0,3.5)$ & 0.10 \\
\hline Cardiac troponin $\mathrm{T}, \mathrm{ng} / \mathrm{L}$ & $3(3,4)$ & $3(3,13)$ & $4(3,13)$ & $12(3,40)$ & $<0.001$ \\
\hline NT-proBNP, ng/L & $74(34,174)$ & $110(54,254)$ & $178(52,607)$ & $491(143,2384)$ & $<0.001$ \\
\hline
\end{tabular}

RAS, renin-angiotensin-system; eGFR, estimated glomerular filtration rate; LDL, low-density lipoprotein; NT-proBNP, N-terminal pro-B-type natriuretic peptide; SN, secretoneurin.

was associated with higher hs-TnT, NT-proBNP, and SN concentrations, as measured by both the ELISA and RIA (Table 2). Greater concentrations of SN ELISA were associated with ACS in unadjusted models (odds ratio: 2.05 [95\% confidence interval [CI]: 1.32-2.78] per log unit increase, $p<0.001)$ with an AUC of $0.66(0.61-0.71)$. Similarly, greater concentrations of SN RIA were associated with ACS in unadjusted models (odds ratio: 0.99 [95\% CI: $0.20-1.79$ ] per $\log$ unit increase, $p=0.014$ ) with AUC 0.59 (0.54-0.65). The diagnostic accuracy of hs-TnT to diagnose ACS patients in our study was an AUC of 0.82 (0.78$0.86)$.

\section{SN in Predicting All-Cause Mortality}

During a mean $6.2 \pm 1.5$-year follow-up, 65 (16\%) of the patients died and $49(75 \%)$ of these were classified with ACS as the cause of the index hospitalization. SN measured by ELISA was associated with time to all-cause mortality (hazard ratio: 2.64 [95\% CI: 1.94-3.60) per log unit increase, $p<0.001$ ), with a corresponding Harrell's C-statistics of 0.72 (95\% CI: 0.65-0.79). The prognostic performance of SN ELISA persisted after adjusting for demographics $(p=0.014)$ and comorbidities $(p=0.03)$ but not after further adjustments for eGFR and hs-TnT ( $p=0.58)$ (Table 3). SN measured by the RIA was also associated with time to all-cause mortality (hazard ratio: 4.13 [95\% CI: 2.15-7.92] per log unit increase, $p<0.001$ ), with a Harrell's C-statistics of 0.64 (95\% CI 0.56-0.72), which was significantly lower than that for SN ELISA ( $p=0.007$, for difference between $\mathrm{SN}$ assays). The prognostic performance of SN RIA was attenuated after adjusting for age and sex and was no longer significant $(p=$ 0.20 ). Survival curves by quartiles of SN measured by ELISA and RIA are presented in Figure 2. 
Table 2. Concentrations of biomarkers in patients with and without ACS

\begin{tabular}{lccr}
\hline & Non-ACS $(n=240)$ & ACS $(n=161)$ & $p$ value \\
\hline SN ELISA, pmol/L & $28.0(24.5,34.0)$ & $32.8(27.5,42.8)$ & $<0.001$ \\
SN RIA, pmol/L & $123(106,143)$ & $134(115,154)$ & 0.001 \\
Cardiac troponin T, ng/L & $3(3.4)$ & $17(5,86)$ & $<0.001$ \\
NT-proBNP, ng/L & $79(31,209)$ & $374(152,1319)$ & $<0.001$ \\
\hline
\end{tabular}

ACS, acute coronary syndrome; SN, secretoneurin; RIA, radioimmunoassay; NTproBNP, N-terminal pro-B-type natriuretic peptide.

Table 3. SN measured by the novel ELISA and the contemporary RIA in association with all-cause mortality

\begin{tabular}{|c|c|c|c|c|c|c|c|c|}
\hline & \multicolumn{2}{|l|}{ Unadjusted } & \multicolumn{2}{|c|}{ Adjusted for age and sex } & \multicolumn{2}{|c|}{$\begin{array}{l}\text { Adjusted for age, sex, and } \\
\text { comorbidities* }\end{array}$} & \multicolumn{2}{|c|}{$\begin{array}{l}\text { Adjusted for age, sex, } \\
\text { comorbidities, }{ }^{*} \text { eGFR, and } \\
\text { cTnT }\end{array}$} \\
\hline SN ELISA & $2.64(1.94-3.60)$ & $<0.001$ & $1.79(1.12-2.85)$ & 0.014 & $1.76(1.06-2.92)$ & 0.03 & $1.21(0.62-2.35)$ & 0.58 \\
\hline SN RIA & $4.12(2.15-7.92)$ & $<0.001$ & $1.89(0.71-5.04)$ & 0.20 & $2.08(0.74-8.83)$ & 0.16 & $1.20(0.43-3.36)$ & 0.73 \\
\hline
\end{tabular}

eGFR, estimated glomerular filtration rate; cTnT, cardiac troponin; SN, secretoneurin; RIA, radioimmunoassay; HR, hazard ratio; CI, confidence interval. *Hypertension, atrial fibrillation, previous myocardial infarction, and heart failure.
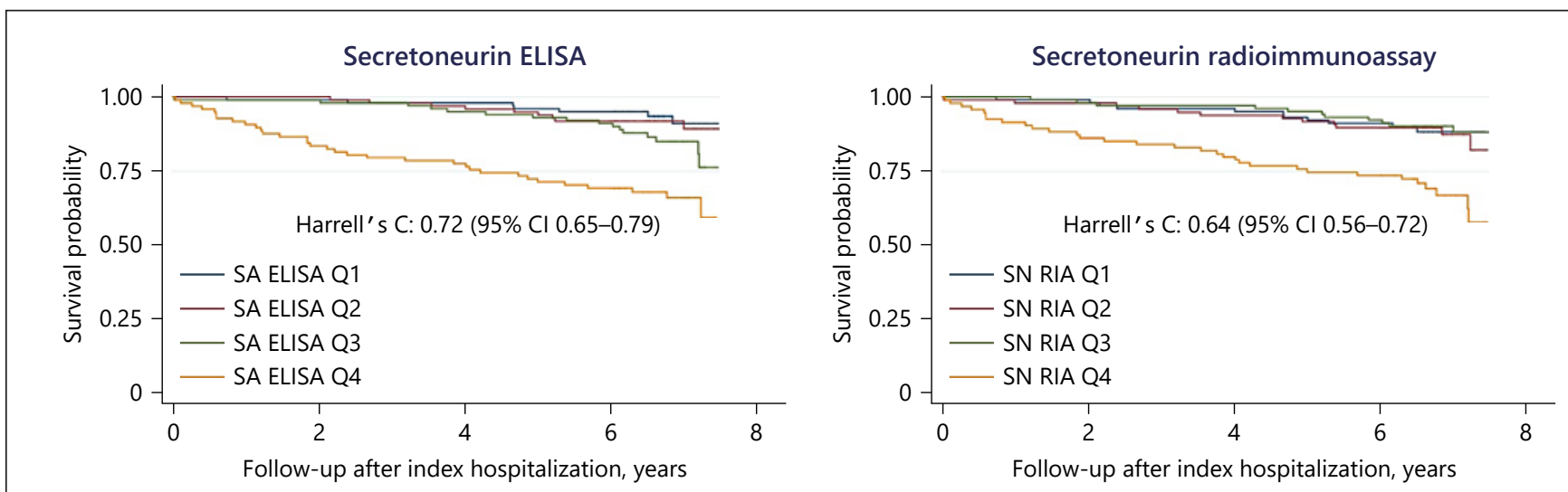

Fig. 2. Survival plots in patients with suspected ACS by concentrations of SN measured by different assays. The Kaplan-Meier survival plot of patients hospitalized with suspected ACS $(n=401)$ by quartiles of SN measured by a novel ELISA (left panel) and a RIA (right panel) with corresponding Harrell's C-statistics. ACS, acute coronary syndrome; SN, secretoneurin; RIA, radioimmunoassay.

\section{Discussion}

We report details of a novel research-use-only SN ELISA that has excellent performance and very good precision across different SN concentrations. SN ELISA also provided superior prognostic information compared to the established SN RIA in unselected patients hospitalized for chest pain.

Novel biomarkers should meet the following 3 key characteristics to have clinical potential: (1) there should be a robust and easily available method for biomarker measurement, (2) the biomarker should provide incre- 
mental information to established risk indices and biomarkers, and (3) the biomarker should influence patient management [22]. So far, SN has been found to provide incremental prognostic information to established risk indices in patients with acute heart failure, ventricular arrhythmia-induced cardiac arrest, cardiovascular-related respiratory failure, cardiac surgical patients $[9,23]$, and in patients with severe infections $[10,11]$. However, these data have all been produced by an in-house SN RIA, which cannot easily be transferred to a routine clinical chemistry laboratory for day-to-day use. Hence, the development of an easy-to-use SN ELISA is a step forward for SN as a candidate cardiovascular biomarker by fulfilling the criteria related to biomarker analysis. This novel method of measuring SN is substantially more time-efficient, and with potential for later conversion to large-scale automated ELISA instruments. Our detailed characterization of the 2 monoclonal sheep antibodies selected for the assay and the excellent performance of SN ELISA across a range of SN concentrations support that this assay will be valuable for future studies on SN as a cardiovascular biomarker. SN concentrations measured with ELISA and RIA demonstrated acceptable correlation, especially considering the long storage of samples between RIA and ELISA analyses. Moreover, as correlation coefficients for SN ELISA and hs-TnT and SN ELISA and NT-proBNP were in the same range, as previously reported for SN RIA [1, 3, 4, 9, 23], we consider SN ELISA an attractive alternative to the established RIA for SN quantification.

SN measurements have previously been found to improve risk assessment across different populations with myocardial dysfunction [1, 3, 4, 8-11]. In contrast, SN concentrations did not improve diagnosis of heart failure over NT-proBNP measurements in unselected patients hospitalized with acute dyspnea [1]. We now extend the data on $\mathrm{SN}$ as a cardiovascular biomarker to a new population of low-risk chest pain patients. In line with the current model of SN as a prognostic cardiovascular biomarker, SN provided prognostic information in patients with chest pain that was independent of demographics and comorbidities but not incremental to cTnT. While SN has been shown to provide prognostic information on top of cTnT and NT-proBNP in other clinical settings [1, 3, 4, 9-11], the central role of these biomarkers in ACS, and the modest statistical power in the current population, may account for the lack of prognostic independence for $\mathrm{SN}$ in this cohort of low-risk chest pain patients. Although SN concentrations were higher in patients with ACS, the diagnostic performance in chest pain patients was limited and not clinically useful. Hence, SN could have prognos- tic potential in low-risk, unselected chest pain patients but is not a candidate biomarker to diagnose acute myocardial injury and necrosis in chest pain patients. Of relevance for this study, the recently developed SN ELISA provided superior prognostic accuracy to the established SN RIA. As demonstrated in Figure 2, patients with the highest SN concentrations (quartile 4) had a poor prognosis with both assays. In contrast, only the SN ELISA seemed to identify high-risk patients also in quartile 3, which could relate to superior precision of the SN ELISA in only slightly elevated SN concentrations. The reported AUC of the SN ELISA to predict mortality in these lowrisk chest pain patients was comparable to AUCs previously reported for the SN RIA in more high-risk patients, such as patients with acute heart failure, cardiovascularrelated respiratory failure, cardiac surgical patients $[9$, $23]$, and with severe infections $[10,11]$. However, whether SN can improve risk assessment over established risk indices and cardiac biomarkers in low-risk chest pain patients will need to be tested in larger cohorts with more events than the current population.

Currently, the pathobiology reflected by high SN concentrations in patients with myocardial dysfunction is not fully established. Although we find higher SN concentrations for chest pain patients with coronary artery disease than patients with non-ACS-related chest pain, there was a large overlap in SN concentrations between these groups as reflected in AUCs of 0.66 and 0.59 for SN ELISA and SN RIA, respectively. The minor impact by acute myocardial ischemia and necrosis on circulating SN concentrations excludes $\mathrm{SN}$ as a diagnostic biomarker in chest pain patients, which is not surprising as $\mathrm{SN}$ is produced in different organs throughout the body [18]. Pertinent to this point, our study supports $\mathrm{SN}$ as an index of cardiac and noncardiac pathobiology during myocardial dysfunction as both eGFR and hs-TnT were independently associated with higher SN concentrations in multivariable linear regression analysis. Still, our study did not find acute myocardial injury and necrosis to represent strong stimuli for high SN concentrations in patients, and therefore, future studies should assess additional cardiac pathobiology as stimuli for high SN concentrations in situations of myocardial dysfunction.

This study has some strengths and limitations. We believe the detailed characterization of the monoclonal sheep antibodies represent a strength for the novel SN ELISA. We have also included unselected patients with chest pain and performed biomarker testing according to standard statistical criteria for diagnostic and prognostic utility, including benchmarking against cardiac biomark- 
ers already in clinical use. Limitations of the current study include a relatively low number of patients in a low-risk cohort, moderate duration of follow-up, and lack of information on the specific cause of death in our patients.

\section{Conclusion}

We provide detailed data on a novel research-use-only SN ELISA with excellent performance and very good precision across a range of SN concentrations. The SN ELISA also demonstrated higher AUC for diagnosis and superior prognostic accuracy compared to the established RIA in unselected chest pain patients, but whether the SN ELISA may provide incremental prognostic information to established risk indices and biomarkers in low-risk chest pain patients will have to be tested in additional studies with larger cohorts and more events.

\section{Acknowledgments}

We thank study personnel and physicians at the Akershus University Hospital that participated in the inclusion of patients in the ACE 3 Study. We are also grateful for help from research coordinator Ronja Hesthammer, Ph.D, Division of Research and Innovation, Akershus University Hospital.

\section{Statement of Ethics}

The current study was approved by the Regional Medical Ethics Committee (\#6.1008.2827) and conducted in accordance with the Helsinki Declaration. All participants provided written informed consent.

\section{Conflict of Interest Statement}

P.L.M. has served on advisory boards for Novartis and Novo Nordisk and has received consulting honoraria from Novartis, AmGen, and Novo Nordisk. M.S., G.C., T.O., and H.R. are partners in a patent regarding the use of $\mathrm{SN}$ as a biomarker in cardiovascular disease and in patients with critical illness. M.S., G.C., A.L.F., T.O., and H.R. are stock owners in CardiNor AS, which holds the license to commercialize secretoneurin. A.H.O., A.L.F, M.S., T.O., A.H.O., and H.R. have also received personal payments from CardiNor AS. T.O. has served on advisory boards and received speaker's honoraria and travel funding from Roche Diagnostics and Roche Diagnostics that provided hs-TnT and NTproBNP kits at a reduced price via the Akershus University Hospital. T.O. and H.R. have received personal fees from Novartis and Thermo Fisher BRAHMS. J.S. and J.P. are employees of Bioventix and Kaivogen, respectively, and both of these companies are involved in production and sale of the SN ELISA. The remaining authors have no disclosures and report no conflicts of interest pertaining to this work.

\section{Funding Sources}

Dr. Myhre is supported by a research grant from the SouthEastern Norway Regional Health Authority to Dr. Røsjø, and Dr. Ottesen is supported by a research grant from the Norwegian Research Council to Dr. Røsjø.

\section{Author Contributions}

P.L.M. performed the clinical analysis from the ACE3 study and drafted the manuscript. A.H.O. performed the experimental SN analysis and drafted the manuscript. A.L.F., J.S., J.P., and M.S. participated in the development of SN ELISA. S.T. and A.D.H. participated in the data collection in the ACE3 study. G.C., T.O., and H.R. designed and supervised the study. All the authors critically reviewed the manuscript.

\section{References}

1 Ottesen AH, Louch WE, Carlson CR, Landsverk OJB, Kurola J, Johansen RF, et al. Secretoneurin is a novel prognostic cardiovascular biomarker associated with cardiomyocyte calcium handling. J Am Coll Cardiol. 2015 Feb 3;65(4):339-51.

2 Bartolomucci A, Possenti R, Mahata SK, Fischer-Colbrie R, Loh YP, Salton SR. The extended granin family: structure, function, and biomedical implications. Endocr Rev. 2011 Dec;32(6):755-97.

3 Myhre PL, Ottesen AH, Okkonen M, Linko R, Stridsberg M, Nygård S, et al. Prognostic value of secretoneurin in patients with acute respiratory failure: data from the FINNALI study. Clin Chem. 2016 Oct;62(10):1380-9.
4 Rosjo H, Stridsberg M, Ottesen AH, Nygard S, Christensen G, Pettila V, et al. Prognostic value of secretoneurin in critically Ill patients with infections. Crit Care Med. 2016 Oct; 44(10):1882-90.

5 Anderson ME. Will secretoneurin be the next big thing? J Am Coll Cardiol. 2015 Feb 3; 65(4):352-4

6 Egger M, Schgoer W, Beer AG, Jeschke J, Leierer J, Theurl M, et al. Hypoxia up-regulates the angiogenic cytokine secretoneurin via an HIF-1alpha- and basic FGF-dependent pathway in muscle cells. FASEB J. 2007;21(11): 2906-17.
7 Theurl M, Schgoer W, Albrecht-Schgoer K, Lener D, Wolf D, Wolf M, et al. Secretoneurin gene therapy improves hind limb and cardiac ischaemia in Apo $\mathrm{E}^{-/-}$mice without influencing systemic atherosclerosis. Cardiovasc Res. 2014;105(1):96-106.

8 Hasslacher J, Lehner GF, Harler U, Beer R, Ulmer H, Kirchmair R, et al. Secretoneurin as a marker for hypoxic brain injury after cardiopulmonary resuscitation. Intensive Care Med. 2014 Oct;40(10):1518-27.

9 Brynildsen J, Myhre PL, Lyngbakken MN, Klaeboe LG, Stridsberg M, Christensen G, et al. Circulating secretoneurin concentrations in patients with moderate to severe aortic stenosis. Clin Biochem. 2019 Sep;71:17-23. 
10 Røsjø H, Masson S, Caironi P, Stridsberg M, Magnoli M, Christensen G, et al. Prognostic value of secretoneurin in patients with severe sepsis and septic shock: data from the albumin Italian outcome sepsis study. Crit Care Med. 2018 May;46(5):e404-e10.

11 Røsjø H, Nygård S, Kaukonen KM, Karlsson S, Stridsberg M, Ruokonen E, et al. Prognostic value of chromogranin A in severe sepsis: data from the FINNSEPSIS study. Intensive Care Med. 2012 May;38(5):820-9.

12 Tveit SH, Myhre PL, Hoff NJS, Le TM, Seljeflot I, Røysland R, et al. Superiority of high sensitivity cardiac troponin T vs. I for long-term prognostic value in patients with chest pain; data from the akershus cardiac examination (ACE) 3 study. Clin Biochem. 2020 Apr;78:10-7.

13 Hamm CW, Bassand JP, Agewall S, Bax J, Boersma E, Bueno $\mathrm{H}$, et al. ESC guidelines for the management of acute coronary syndromes in patients presenting without persistent ST-segment elevation: the task force for the management of acute coronary syndromes (ACS) in patients presenting without persistent ST-segment elevation of the European Society of Cardiology (ESC). Eur Heart J. 2011 Dec;32(23):2999-3054.
14 Thygesen K, Alpert JS, Jaffe AS, Simoons ML, Chaitman BR, White HD, et al. Third universal definition of myocardial infarction. J Am Coll Cardiol. 2012 Oct 16;60(16): 1581-98.

15 Roffi M, Patrono C, Collet JP, Mueller C, Valgimigli M, Andreotti F, et al. 2015 ESC guidelines for the management of acute coronary syndromes in patients presenting without persistent ST-segment elevation: task force for the management of acute coronary syndromes in patients presenting without persistent ST-segment elevation of the European Society of Cardiology (ESC). Eur Heart J. 2016 Jan 14;37(3):267-315.

16 Levey AS, Stevens LA, Schmid CH, Zhang YL, Castro AF3rd, Feldman HI, et al. A new equation to estimate glomerular filtration rate. Ann Intern Med. 2009 May 5;150(9):604-12.

17 Stridsberg M, Eriksson B, Janson ET. Measurements of secretogranins II, III, V and proconvertases $1 / 3$ and 2 in plasma from patients with neuroendocrine tumours. Regul Pept. 2008 Jun 5;148(1-3):95-8.

18 Rosjo H, Stridsberg M, Florholmen G, Stenslokken KO, Ottesen AH, Sjaastad I, et al. Secretogranin II; a protein increased in the myocardium and circulation in heart failure with cardioprotective properties. PLoS One. 2012;7(5):e37401.

19 Myhre PL, Ottesen AH, Okkonen M, Linko R, Stridsberg M, Nygard S, et al. Prognostic value of secretoneurin in patients with acute respiratory failure: data from the FINNALI Study. Clin Chem. 2016;62(10):1380-9.

20 Røsjø H, Husberg C, Dahl MB, Stridsberg M, Sjaastad I, Finsen AV, et al. Chromogranin B in heart failure: a putative cardiac biomarker expressed in the failing myocardium. Circ Heart Fail. 2010 Jul;3(4):503-11.

21 Frank R, Overwin H. SPOT synthesis. Epitope analysis with arrays of synthetic peptides prepared on cellulose membranes. Methods $\mathrm{Mol}$ Biol. 1996;66:149-69.

22 Morrow DA, de Lemos JA. Benchmarks for the assessment of novel cardiovascular biomarkers. Circulation. 2007 Feb 27;115(8): 949-52.

23 Brynildsen J, Petäjä L, Myhre PL, Lyngbakken MN, Nygård S, Stridsberg M, et al. Circulating secretoneurin concentrations after cardiac surgery: data from the FINNish acute kidney injury heart study. Crit Care Med. 2019 May; 47(5):e412-e19. 